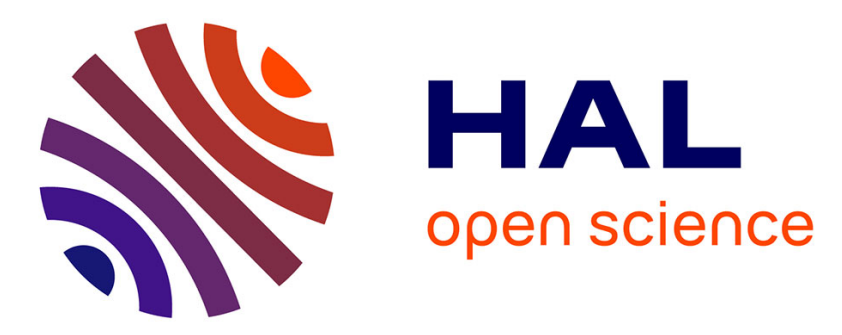

\title{
Diaphorina citri (Kuwayama) en Guadeloupe, premier signalement pour les Caraïbes (Hem., Psyllidae)
}

\author{
D. Burkhardt, C. Grapin
}

\section{To cite this version:}

D. Burkhardt, C. Grapin. Diaphorina citri (Kuwayama) en Guadeloupe, premier signalement pour les Caraïbes (Hem., Psyllidae). Bulletin de la Société Entomologique de France, 1998, 103 (1), pp.32. hal-02691871

\section{HAL Id: hal-02691871 \\ https://hal.inrae.fr/hal-02691871}

Submitted on 1 Jun 2020

HAL is a multi-disciplinary open access archive for the deposit and dissemination of scientific research documents, whether they are published or not. The documents may come from teaching and research institutions in France or abroad, or from public or private research centers.
L'archive ouverte pluridisciplinaire HAL, est destinée au dépôt et à la diffusion de documents scientifiques de niveau recherche, publiés ou non, émanant des établissements d'enseignement et de recherche français ou étrangers, des laboratoires publics ou privés. 
Jean ETIENNE, Danicl BURCKHARDT et Cyril GRAPIN. - Diaphorina citri (Kuwayama) en Guadeloupe, premier signalement pour les Caraïbes (Hem., Psyllidae).

Dans la Région Néotropicale Diaphorina citri n'était signalé jusqu’à présent que du Brésil (Commonwealth Institute of Entomology, 1974), du Honduras (Burckhardt et Martinez, 1989) et d'Uruguay (Bernal, 1991). Il vient d'être découvert en Guadeloupe en Janvier 1998 dans deux Jardins créoles l'un à Baie-Mahault- La Jaille et l'autre à LamentinBréfort. Dans ces deux jardins adultes et larves étaient présents en abondance sur jeunes pousses d'orangers, de mandariniers et de tangelos. Aucune trace de parasitisme n'a pu être mise en évidence à partir des échantillons prélevés.

Diaphorina citri se développe uniquement sur des Rutaceae et est particulièrement nuisible aux plantations de citrus car il est un des deux psylles vecteurs de la grave maladie du " greening " (Burckhardt, 1994). Cette maladie, qui provoque un déclin rapide des plantations, n'est pas d'origine mycoplasmique (Aubert et al.,1980) mais est dûe à une bactérie intracellulaire qui affecte principalement le phloeme des parties tendres de l'arbre et seuls les genres Citrus, Fortunella et Poncirus semblent susceptibles de la multiplier dans leurs tissus (Grisoni et al., 1993).

En Guadeloupe des prospections systématiques devraient être effectuées sur les 3 genres précités ainsi que sur le genre Murraya plante hôte naturelle probable de ce psylle. Parallelement des recherches sur la présence du "greening" sont à réaliser (greffes d'inoculation sur plants sensibles, mise en évidence de la bactérie par microscopie électronique) car cette maladie peut être disséminée très facilement à partir de greffage d'écussons provenant de plants contaminés ou par la transmission naturelle de psylles.

Seule une bonne connaissance de la distribution du vecteur et la localisation d'éventuels foyers de la maladie permettra de prendre des mesures concrêtes pour la protection des plantations citricoles. Ces mesures existent et ont été mises en place notamment à l'Ile de la Réunion et à l'Ile Maurice qui sont actuellement les deux seuls pays reconnus comme ayant à la fois la maladie du "greening" et les deux espèces de psylles vecteurs. En effet les introductions des Eulophidae parasites spécifiques des psylles Trioza erytreae (Del Guercio) (Etienne, 1978) et Diaphorina citri (Etienne et Aubert, 1980) ont permis, associées à la diffusion de matériel végétal sain, de surmonter l'obstacle que présentait le "greening" pour la culture de Citrus dans ces deux îles.

En Guadeloupe l'introduction de Tamarixia radiata (Waterston) parasite spécifique de Diaphorina citri, à partir de la Réunion, est donc à envisager pour limiter ce psylle et éviter ainsi une explosion de ses populations dans un proche avenir.

Enfin compte tenu de l'importance agronomique de Diaphorina citri pour l'avenir économique des plantations de Citrus de la région, il paraît fondamental de rechercher activement des à présent ce psylle dans les pays ayant d'importantes superficies cultivées (Cuba, Haiti, Jamaïque, Porto Rico, République Dominicaine, et la Floride ) afin qu'en cas de découverte, ces pays puissent prendre très vite les mesures qui s'imposent.

\section{AUTEURS CITES}

AUBERT B., BOVE J.M. \& ETIENNE J., 1980. - La lutte contre la maladie du "greening" des agrumes à l'île de la Réunion. Résultats et perspectives. Fruits 35 (10): 605-624.

BERNAL R., 1991. - Diaphorina citri (Homoptera : Psyllidae) Nuevo insecto detectado en montes citricos en el area de Salto, Uruguay. INIA, Uruguay, Hocha de divulgacion 25: 1-2.

BURCKHARDT D., 1994. - Psylloid pests of temperate and subtropical crop and ornamental plants (Hemiptera, Psylloidea): a review. Trends in Agricultural Sciences, Entomology 2: 173-186.

BURCKHARDT D. \& MARTINEZ M., 1989 . - Note sur la présence au Honduras d'un redoutable ennemi des Citrus : Diaphorina citri Kuwayama (Hom. Psylloidea Psyllidae). Bulletin de la Société entomologique de France 94 (1-2): 6566.

Commonwealth Institute of Entomology, 1974 - Distrib. Maps Pests, Ser. A (Agricultural) n³34.

ETIENNE J., 1978. - Contrôle biologique à la Réunion de Trioza erytreae (Homopt. Psyllidae) au moyen de Tetrastichus dryi (Hym. Eulophidae). Fruits 33 (12) : 877-882.

ETIENNE J. \& AUBERT B., 1980. - Biological control of Psyllid vectors of Greening disease on Reunion Island. In : E.C. CAVALAN, S.M. GARNSEY, L.W. Timmer ed.Proceeding of the Eighth Conf. Intern. Org. Citrus Virologists, $1980: 118-$
121.

GRISONI M., 1993. - La culture des agrumes à l'île de la Réunion, CIRAD-FLHOR, 102p.

(INRA - URPV, Lab. d'Entomologie Lutte Intégrée, B.P. 515, F-97165 Pointe-à-Pitre Cedex, Guadeloupe, F.W.I. ; Muséum d'Histoire Naturelle, Augustinergasse 2, postfach, 4001, Basel, Suisse). 